\title{
Terra Firma-Forme Dermatosis
}

\author{
Dermatose Terra Firma-Forme
}

\author{
Adelina COSTIN $\triangle^{1}$, Constança FURTADO ${ }^{1}$ \\ Acta Med Port 2020 May;33(5):350-350 - https://doi.org/10.20344/amp.11847
}

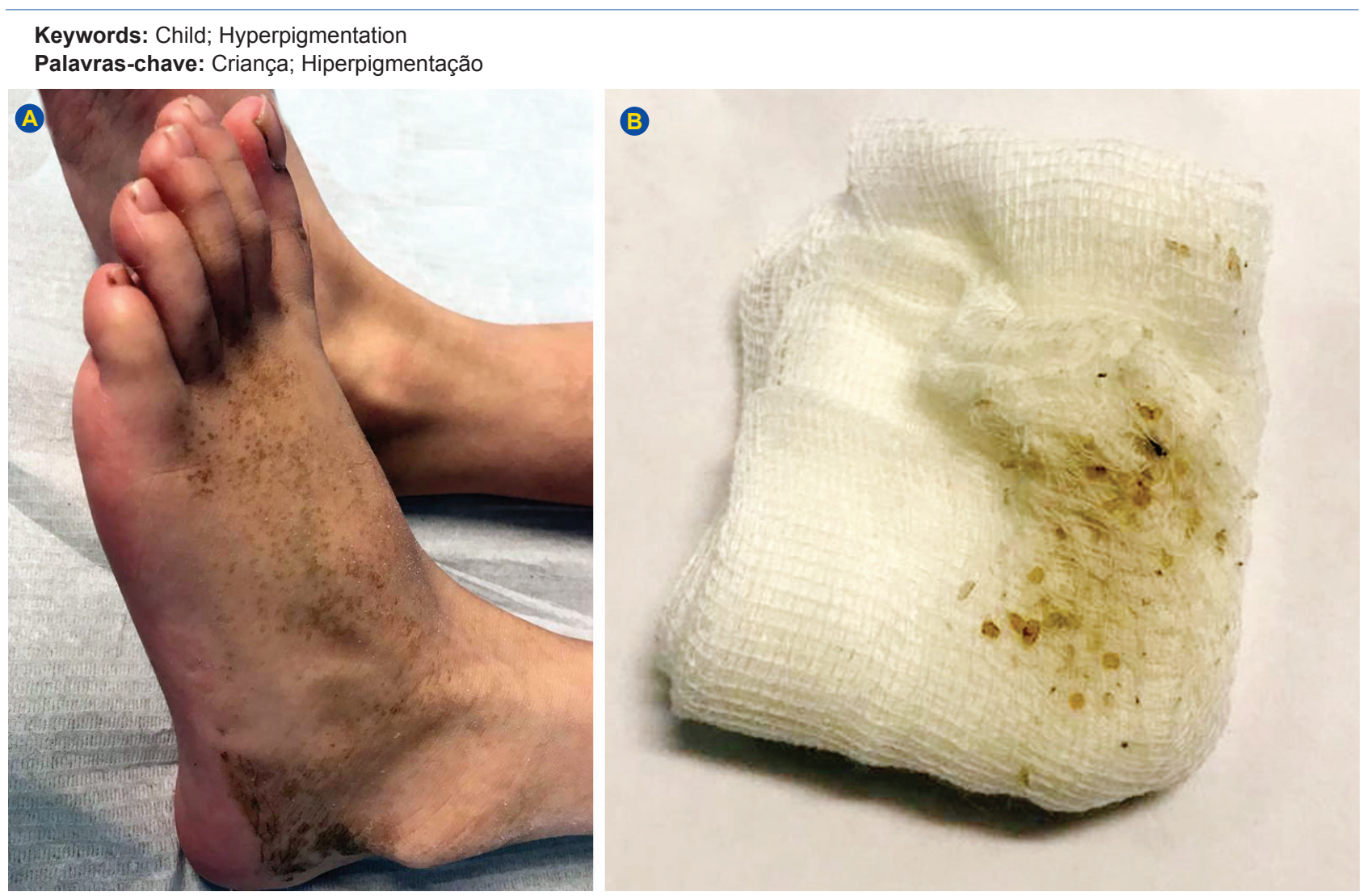

Figure 1 - (A, B) The wipe test: easy removal of the verrucous lesion by wiping with an alcohol-soaked pad

A 10-year-old boy with a history of atopy was referred to the outpatient dermatology clinic with long-standing brownish verrucous plaques with a 'dirt'-like appearance on the glabellar region, posterior neck, superior trunk and lower extremities including peri-malleolar and dorsal feet.

Terra firma-forme dermatosis is an idiopathic acquired retention hyperkeratosis with asymptomatic brown plaques that can easily be removed with isopropyl alcohol rubbing, the so-called wipe test. ${ }^{1}$ Although frequently described in children, it is also common in adults and older individuals. The main differential diagnoses are dermatitis neglecta, confluent and reticulated papillomatosis, acanthosis nigricans and the 'dirty neck syndrome' of atopic dermatitis.

It is important to recognize this benign and easily confirmed dermatosis in order to prevent unnecessary work-up and treatments.

PROTECTION OF HUMANS AND ANIMALS: The authors declare that the procedures were followed according to the regulations established by the Clinical Research and Ethics Committee and to the Helsinki Declaration of the World Medical Association.

DATA CONFIDENTIALITY: The authors declare having followed the protocols in use at their working center regarding patients' data publication. INFORMED CONSENT: Obtained.

CONFLICTS OF INTEREST: All authors report no conflict of interest.

FUNDING SOURCES: The authors declare that there were no external sources of study for the performance of this article.

\section{REFERENCES}

1. Berk DR. Terra firma-forme dermatosis: a retrospective review of 31 patients. Pediatr Dermatol. 2012;29:297-300.

\footnotetext{
1. Dermatology and Venereology Department. Hospital Garcia de Orta. Almada. Portugal.

$\triangle$ Autor correspondente: Adelina Costin. adelina_costin@hotmail.com

Recebido: 23 de janeiro de 2019 - Aceite: 17 de junho de 2019 | Copyright @ Ordem dos Médicos 2020
} 